Original Article

\title{
Relationship between agility and lower limb muscle strength, targeting university badminton players
}

TAKUYa Sonoda, RPT ${ }^{1)}$, Yuto Tashiro, RPT, MS ${ }^{1)}$, Yusuke SuzUki, RPT, MS ${ }^{1)}$, Yu Kajiwara, RPT, MS ${ }^{1,2)}$, Hala ZEIDAN, RPT, MS ${ }^{1)}$, Yuki YoKota, RPT ${ }^{1)}$, Mirei Kawagoe, RPT ${ }^{1}$, Yasuaki Nakayama, RPT ${ }^{1)}$, Tsubasa Bito, RPT ${ }^{1)}$, Kanako Shimoura, RPT ${ }^{1)}$, Masataka Tatsumi, RPT ${ }^{1)}$, Kengo NaKai, RPT ${ }^{1)}$, Yuichi Nishida, RPT ${ }^{1)}$, SoYoKa Yoshimi, RPT ${ }^{1)}$, Tomoki AOYAMA, MD, $\mathrm{PhD}^{1)^{*}}$

1) Department of Physical Therapy, Human Health Science, Graduate School of Medicine, Kyoto University: 53 Kawahara-cho, Shogoin, Sakyo-ku, Kyoto 606-8507, Japan

2) Department of Physical Therapy, Faculty of Health Science, Kio University, Japan

\begin{abstract}
Purpose] Targeting university badminton players, this study investigated the relationship between agility, which is associated with performance in badminton, and lower limb muscle strength, and examined which muscles influence agility. [Subjects and Methods] A total of 23 male university badminton players were evaluated for side-shuffle test scores and lower limb strength. The relationships between agility, lower limb strength, and duration of experience playing badminton were evaluated using a correlation analysis. Moreover, the relationship between agility and lower limb strength was evaluated by partial correlation analysis, adjusting for the effects of experience of each badminton player. [Results] The agility score correlated with hip extension and ankle plantar flexion strength, with adjustment for badminton experience. [Conclusion] This study suggests that hip extension training and improvement in ankle plantar flexion strength may improve agility.

Key words: Badminton, Agility, Muscle strength
\end{abstract}

(This article was submitted Oct. 23, 2017, and was accepted Nov. 28, 2017)

\section{INTRODUCTION}

Badminton is one of the most popular sports in the world ${ }^{1)}$. It is a racquet sport played by individual opposing players (singles) or pairs of opposing players (doubles), who take positions on opposite halves of a rectangular court that is divided by a net ${ }^{2}$. In badminton rallies, players hit the shuttlecocks back and forth to move the opponents and to win the rallies, so players require quick steps to return the shuttlecocks at various speed in various directions ${ }^{3)}$. Badminton players must repeat actions in a short time with high speed and intensity within $80 \mathrm{~m}^{2}$ court $^{4}$ ). Therefore, badminton players need great agility. Tiwari et al. showed that agility was associated with performance during a badminton match ${ }^{2}$.

Agility is defined as a rapid movement of the entire body with a change in velocity or direction in response to a stimulus, such as the shuttlecock which an opponent player has hit ${ }^{5)}$. The change in velocity is the ability to accelerate and decelerate concurrent with a change in direction. Young et al. showed that this ability consisted of several factors such as straight sprint, leg muscle strength and running technique, demonstrating that agility requires muscle power in order to move quickly and technique to move efficiently ${ }^{6}$. Milanović et al. reported that specific training involving speed, agility and quickness improved agility ${ }^{7}$. In relation to muscle strength, a study of 8 weeks of jump squat training also reported an improvement in agility $^{8)}$. By contrast, another study showed that three sets of three repetitions of heavy squats at $90 \%$ of the one-repetition

*Corresponding author. Tomoki Aoyama (E-mail: blue@hs.med.kyoto-u.ac.jp)

(C2018 The Society of Physical Therapy Science. Published by IPEC Inc.

(c) (1) $\odot$ This is an open-access article distributed under the terms of the Creative Commons Attribution Non-Commercial No Deriva-

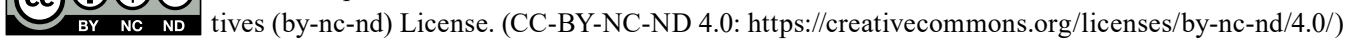


maximum did not contribute to an improvement in agility ${ }^{9}$. There is no common opinion regarding the relationship between agility and lower limb strength and which muscles contribute to agility. Identifying this relationship may contribute to efficient agility training.

Therefore, the purpose of this study was to investigate the relationship between agility which is associated with performance in badminton, and lower limbs muscle strength, and to examine which muscles influence agility.

\section{SUBJECTS AND METHODS}

A total of 23 male university badminton players (mean age $=20.2 \pm 1.0$ years, mean weight $=60.5 \pm 5.4 \mathrm{~kg}$, mean height $=170.7 \pm 6.1 \mathrm{~cm}$, mean badminton experience $=5.0 \pm 3.1$ years) without pain in the lower limbs participated in this study (Table 1). Duration of badminton experience meant the experience until this experiment. This study was approved by Kyoto university ethics committee (R0639) and obtained informed consent. Data regarding agility and lower limbs strength were collected.

Agility data were collected using the side-shuffle test, the most commonly used agility test in Japan. Subjects used their own shoes and had adequate warm-up before the test. It was referred to in the Japanese New physical fitness test of the Ministry of Education, Culture, Sports, Technology ${ }^{10)}$. A tester drew three lines one meter apart. Subjects stood on the center line and when told to start, they took side steps to the right line until they stepped on or across the line. This was then repeated for the left line. This exercise was performed for 20 seconds on each side and a point was scored for each time the subjects passed the lines. The entire measurement was performed twice and the highest score was used.

Lower limb strength measurements were performed on each side twice by an experienced measurer. The average of the maximum right and left strength was regarded as the representative value of lower limb strength. Subjects had enough warmup before the measurements. Subjects crossed their arms in front of the chest to prevent compensatory motion. Hip flexion/ extension isometric strength and ankle plantar/dorsal flexion isometric strength were measured using the Biodex System4 (Biodex, New York, USA) in a spine position, with hip and knee joints at $90^{\circ}$ flexion and fixing the other side femur, pelvis and trunk. Ankle plantar/dorsal flexion isometric strength were measured sitting in a chair, with the knee joint fully extended and fixing measurement side femur, pelvis and trunk. Knee flexion/extension isometric strength was measured using the IsoforceGT-330 (OG-wellness, Okayama, Japan), at chair sitting position, with the knee joint at $60^{\circ}$ flexion and fixing femurs and pelvis. The evaluator measured the distance from the center of the patella to the top of the medial malleolus and lateral malleolus and calculated the torque.

Data were analyzed using SPSS version 20.0 (IBM Corp, Armonk, New York). The relationships between agility, lower limbs strength and duration of badminton experience were evaluated using a correlation analysis (correlation coefficient $=\mathrm{r}$ ). Moreover, the relationship between agility and lower limb strength was evaluated by partial correlation analysis, adjusting for the effects of badminton experience. Statistical significance was set at $\mathrm{p}<0.05$.

\section{RESULTS}

All participants basic information is presented in Table 1. Data regarding agility measurements and lower limb strength are presented in Table 2. The results of the correlation coefficient analyses between side-shuffle score and duration of badminton experience and lower limb strength are presented in Table 3 . Agility correlated with experience $(r=0.62, p<0.01)$, hip flexion strength $(\mathrm{r}=0.45, \mathrm{p}=0.03)$, hip extension strength $(\mathrm{r}=0.68, \mathrm{p}<0.01)$, knee flexion strength $(\mathrm{r}=0.49, \mathrm{p}=0.03)$ and ankle plantar flexion strength $(\mathrm{r}=0.69, \mathrm{p}<0.01)$. The results of the partial correlation analyses between agility and lower limbs strength are presented in Table 4. Agility correlated with hip extension strength $(\mathrm{r}=0.60, \mathrm{p}<0.01)$ and plantar flexion $(\mathrm{r}=0.70, \mathrm{p}<0.01)$, with adjustment for the effects of badminton experience.

\section{DISCUSSION}

This study indicated that the number of years of badminton experience and side-shuffle score were correlated. This study also indicated that badminton players with the best overall agility had greater hip extension and ankle plantar flexion strength.

Regarding the relationship between years of experience and side-shuffle score, badminton players routinely perform quick steps and changes of direction in training and in matches. In this study, badminton players had a higher score (60.4 points) compared with the general population average score $(50.5 \text { points })^{11)}$. It was suggested that badminton players routinely react with quick side-steps and change in direction, which is likely to improve agility. Many reports showed that specific training is required to improve agility ${ }^{7}, 12$. Therefore, the experience of playing badminton may be correlated with agility as the players undergo specific training for a long time.

Hip extension and ankle plantar flexion strength were correlated to the side-shuffle score in the partial analysis, with adjustment for badminton experience. Shimokochi stated that for a quick change of direction, it is important to lower the center of mass and extend the hip joint quickly and kick the ground ${ }^{13)}$. It was suggested that players with greater hip extension strength can kick the ground strongly and perform quick changes of direction, thereby getting high side-shuffle score. Furthermore, badminton players routinely lower their center of mass in order to move to the spot where the shuttlecock falls. 
Table 1. Characteristics of the subjects $($ mean $\pm \mathrm{SD})$

\begin{tabular}{lr}
\hline Age (years) & $20.2 \pm 1.0$ \\
Height $(\mathrm{cm})$ & $170.7 \pm 6.1$ \\
Weight $(\mathrm{kg})$ & $60.5 \pm 5.4$ \\
Experience (years) & $5.0 \pm 3.1$ \\
\hline
\end{tabular}

SD: standard deviation.
Table 2. Measurement results for agility scores and lower limb strength (mean \pm SD)

\begin{tabular}{lc}
\hline Variables & mean \pm SD \\
\hline Side-shuffle score (points) & $60.4 \pm 5.8$ \\
Hip flexion $(\mathrm{Nm})$ & $65.2 \pm 19.4$ \\
Hip extension $(\mathrm{Nm})$ & $278.5 \pm 72.1$ \\
Knee flexion $(\mathrm{Nm})$ & $97.4 \pm 24.1$ \\
Knee extension $(\mathrm{Nm})$ & $188.0 \pm 42.5$ \\
Ankle plantar flexion $(\mathrm{Nm})$ & $126.0 \pm 28.9$ \\
Ankle dorsal flexion $(\mathrm{Nm})$ & $10.5 \pm 5.4$ \\
\hline
\end{tabular}

SD: standard deviation.

Table 4. Partial correlation coefficient analyses between side shuffle score and lower limb strength, with adjusting for the effects of duration of badminton experience score and duration of badminton experience or lower limb strength

\begin{tabular}{lcc}
\hline Independent variables & $\begin{array}{c}\text { Coefficient of } \\
\text { correlation (r) }\end{array}$ & p-value \\
\hline Experience & 0.62 & $<0.01^{* *}$ \\
Hip flexion & 0.45 & $0.03^{*}$ \\
Hip extension & 0.68 & $<0.01^{* *}$ \\
Knee flexion & 0.49 & $0.03^{*}$ \\
Knee extension & 0.32 & 0.13 \\
Ankle plantar flexion & 0.69 & $<0.01^{* *}$ \\
Ankle dorsal flexion & 0.18 & 0.42 \\
\hline
\end{tabular}

$* \mathrm{p}<0.05, * * \mathrm{p}<0.01$.

Table 3. Correlation coefficient analyses between side-shuffle
To lower the center of mass, many badminton players both squat and maintain their position. Simultaneously maintaining position and squatting may develop hip extension strength because it has been shown that gluteus maximus muscle activity is important for hip extension, and is also correlated with the squat ${ }^{14)}$. Therefore, badminton players tend to develop hip extension strength and therefore may get higher agility scores than average.

Prior research has reported that the ankle plantar flexion muscle provides propulsion during toe-off, helps absorb impact before touchdown, contributes to jump height ${ }^{15,16)}$. This may suggest that strong plantar flexors are more effective for kicking the ground. To kick the ground strongly and laterally may contribute to production of propulsion during toe-off and to a fast side-step. In brief, the players with greater ankle plantar flexion can perform fast side-step and efficiently change direction; therefore, this score may be correlated to ankle plantar flexion strength.

A limitation of this study was that it was unknown when muscles which related to agility contributed to agility. It is necessary to investigate when muscles fire in side-shuffle using an electromyogram, force-plate, three-dimensional motion capture systems and so on because side-shuffle includes many factors such as side-step and change in direction movement.

This study showed that hip extension and ankle plantar flexion strength were correlated to the side-shuffle score regardless of the duration badminton experience. It suggests that hip extension affects the speed of direction and ankle plantar flexion affects side-step speed and change of direction. In the future, an intervention study that shows whether hip extension and ankle plantar flexion strength training develop agility should be performed.

\section{Conflict of interest}

None.

\section{REFERENCES}

1) Lees A: Science and the major racket sports: a review. J Sports Sci, 2003, 21: 707-732. [Medline] [CrossRef]

2) Tiwari LM, Rai V, Srinet S: Relationship of selected motor fitness components with the performance of badminton player. Asian J Phys Educ Comput Sci Sports, 2011, 5: 88

3) Masu Y, Nagai M: Characteristics of lower limb muscle activity during upper limb elevation in badminton players. J Phys Ther Sci, 2016, 28: 2510-2514. 
[Medline] [CrossRef]

4) Cabello Manrique D, González-Badillo JJ: Analysis of the characteristics of competitive badminton. Br J Sports Med, 2003, 37: 62-66. [Medline] [CrossRef]

5) Sheppard JM, Young WB: Agility literature review: classifications, training and testing. J Sports Sci, 2006, 24: 919-932. [Medline] [CrossRef]

6) Young WB, Dawson B, Henry GJ: Agility and change-of direction speed are independent skills: implications for training for agility in invasion sports. Int J Sports Sci Coaching, 2015, 10: 159-169. [CrossRef]

7) Milanović Z, Sporiš G, Trajković N, et al.: Effects of a 12 week SAQ training programme on agility with and without the ball among young soccer players. J Sports Sci Med, 2013, 12: 97-103. [Medline]

8) McBride JM, Triplett-McBride T, Davie A, et al.: The effect of heavy- vs. light-load jump squats on the development of strength, power, and speed. J Strength Cond Res, 2002, 16: 75-82. [Medline]

9) Jullien H, Bisch C, Largouët N, et al.: Does a short period of lower limb strength training improve performance in field-based tests of running and agility in young professional soccer players? J Strength Cond Res, 2008, 22: 404-411. [Medline] [CrossRef]

10) Japanese Ministry of Education, Culture, Sports, Science and Technology: New physical fitness test guidelines http://www.mext.go.jp/component/a_menu/ sports/detail/_icsFiles/afieldfile/2010/07/30/1295079_03.pdf (Accessed Jul. 11, 2017)

11) Japanese Ministry of Education, Culture, Sports, Science and Technology., http://www.mext.go.jp/b menu/toukei/001/022/tk0022/kiso data.htm (Accessed Jul. 11, 2017)

12) Chaalali A, Rouissi M, Chtara M, et al.: Agility training in young elite soccer players: promising results compared to change of direction drills. Biol Sport, 2016, 33: 345-351. [Medline] [CrossRef]

13) Shimokochi Y, Ide D, Kokubu M, et al.: Relationships among performance of lateral cutting maneuver from lateral sliding and hip extension and abduction motions, ground reaction force, and body center of mass height. J Strength Cond Res, 2013, 27: 1851-1860. [Medline] [CrossRef]

14) Yavuz HU, Erdag D: Kinematic and electromyographic activity changes during back squat with submaximal and maximal loading. Appl Bionics and Biomech, 2017 article ID 9084725/http://doi.org/10.aa55/2017/9084725.

15) Neptune RR, Wright IC, van den Bogert AJ: Muscle coordination and function during cutting movements. Med Sci Sports Exerc, 1999, 31: 294-302. [Medline] [CrossRef]

16) Chang E, Norcross MF, Johnson ST, et al.: Relationships between explosive and maximal triple extensor muscle performance and vertical jump height. J Strength Cond Res, 2015, 29: 545-551. [Medline] [CrossRef] 